\title{
Experimental implementation of direct laser alloying
}

\author{
Anton Vladimirovich Sotov ${ }^{1}$, Anton Vasilevich Agapovichev ${ }^{1,{ }^{*}}$ and Vitaly Genadievich Smelov ${ }^{1}$ \\ ${ }^{1}$ Samara University, 443086 Moskovskoye shosse 34, Russia
}

\begin{abstract}
A laser beam, as a source of energy, has been lately widely used in additive technologies for the manufacture of parts for the power engineering industry. One of the promising methods of additive manufacturing based on laser overlay welding of metal wires is the so-called Laser Engineering Net Shape. This work studies the structure and mechanical properties of the sample manufactured using a direct laser alloying unit designed for 07X18H10T stainless steel grade alloying. The allocation map of chemical elements has been examined in this work.
\end{abstract}

\section{Introduction}

The current trend for customization of products and reduction of time to market results in the increased demand from the industry for agile manufacturing technologies. These technologies should be robust and efficient from the standpoint of resources used and they should allow manufacturing quality products. The use of additive technologies enables us to ensure high level of production flexibility in relation to complex design features, while also enable us to directly manufacture parts using CAD models and without machining which results in saving time and resources [1,2].

Lately, in the aviation engine industry great emphasis has been placed on the technologies of additive manufacture using a metal base. This is due to the possibility of manufacturing reduced-weight (bionic) designs, which allows to increase weight-carrying capacity, reduce fuel consumption, and the level of atmospheric emission of pollutants [3-6].

Additive technologies (AT) are the technologies that allow for manufacture of an object using its digital model by way of layer-by-layer application of a material. These technologies may be grouped into two major groups depending on the method of material feeding: (1) Laser Metal Fusion (LMF) features layer-by-layer application of material and includes the technologies of selecting laser sintering (SLS), selecting laser melting (SLM), as well as electron-beam melting (EBM); (2) Laser Metal Deposition (LMD) directly feeds material to the treatment area, and this group includes the technologies of direct metal deposition (DMD), as well as the technology of Laser Engineered Net Shaping (LENS) [7,8].

LMF technology employs a layer-by-layer method of application of material (Figure 1). This method is based on three repeating stages. At the beginning, a thin layer of metal powder is placed on the platform using a special dosing device. At the second stage, a focused laser beam selectively fuses the powder layer, which is placed on the platform. And, at the third stage, the platform is lowered at the thickness of the layer, after which the cycle is repeated. Normally the thickness of a pad is anywhere from $30 \mu \mathrm{m}$ to $50 \mu \mathrm{m}$ therefore manufacture of whole parts may take alloying of thousands of pads.

During LMD process a fused pool is formed on the surface using a laser beam. At the same time, metal powder filler is fed into the fused pool. After hardening the filler forms single welded objects. Several welded objects located next to each other form layers or volumes.

The use of a powder pool in LMF technology enables to synthesize parts with complex geometric shapes. The disadvantages of LMF include its low process speed, relatively small size of the parts manufactured, and significant restrictions as to the use of various powder materials during the process of making parts. LMD technology, on the contrary, exercises higher speeds of generating parts with the possibility of changing powders during the process. However, LMD technology has significant limitations as to the complexity of geometric shapes of the parts manufactured.

LMD technology can be classified into two types depending on the material used: Powder-based (LENS-p) and wire-based (LENS-w) [10-12]. The use of wire materials for generation (growing) of parts has a number of advantages over the powder-based type, namely, higher speed of building of an object, lower cost of materials [13]. Such advantages allow the wire-based technologies to be competitive as compared to the powder-based ones. This work demonstrates an experimental test of the structural layout of a unit that employs LENS-w wire-based technology.

\section{Materials and experimental methods}

The samples have been manufactured using a laser technological installation which included a system of wire feeding, a system of moving the laser head, an

* Corresponding author: agapovichev5@mail.ru 
optical system of observation of the process of laser treatment. Fig. 2 shows the scheme of implementation of the process of making the samples using LENS-w method [14]. Samples for testing were manufactured on the equipment of CAM technology common use center (RFMEFI59314X0003).

In order to test the structural layout of the direct laser alloying unit they have manufactured samples made of 07X18H10T grade stainless steel wire.

Laser overlay of the samples has been performed on a platform in the medium of argon shielding gas. Once manufactured, the samples were separated from the platform using the method of electroerosive machining.

The study of microstructure of the samples has been conducted using Tescan Vega scanning-electron microscope. The study of chemical composition of the samples has been conducted using the energy-dispersive add-on unit of Tascan Vega scanning-electron microscope.

The study of mechanical properties of the samples has been conducted using INSTRON 8802 floor standing system for fatigue testing. Based on a series of preliminary experiments they have identified the modes for impulse laser irradiation used for the samples manufacture.

\subsection{Description of the unit}

The laser technological installation includes a solid-state laser with an irradiation wave length of $1.06 \mu \mathrm{m}$ and with adjustable length of an irradiation impulse from 0.2 $\mathrm{ms}$ to $20 \mathrm{~ms}$, the frequency of tracking the irradiation impulse from $1 \mathrm{~Hz}$ to $20 \mathrm{~Hz}$, the diameter of a focused beam from $0.2 \mathrm{~mm}$ to $5 \mathrm{~mm}$. The system of feeding the material allows for delivering a metal wire to the treatment area at the angle of 45 degrees relative to the laser irradiation. The diameter of the wire used is 0.4 $\mathrm{mm}$. The system of movement steers the laser beam unit in relation to the surface of the sample synthesized in the direction of $\mathrm{X}, \mathrm{Y}$, and $\mathrm{Z}$ axes. The buildup platform remains steady throughout the entire process of generation of geometry. The optical observation system is a binocular with a shutter and lens. The cutoff filters which are built into the system ensure protection of the eyes of an operator from the impact of reflected and dissipated irradiation. The scheme of the experimental system LENS-w technology is presented in Fig. 1.

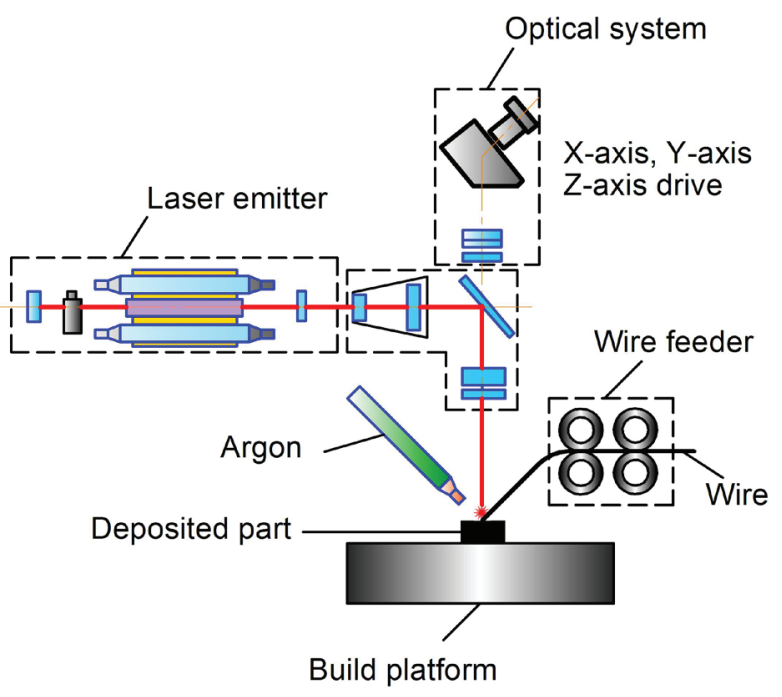

Fig. 1. The schematic of experiment LENS-w system.

The thickness of the build layer was $0.2 \mathrm{~mm}$, which corresponds to half the diameter of the filler wire. The process of implementing LENS-w technology includes the following main steps: supplying protective gas to the treatment zone, melting of the wire by laser radiation, raising the laser head unit to the thickness of the build layer (Fig. 2).

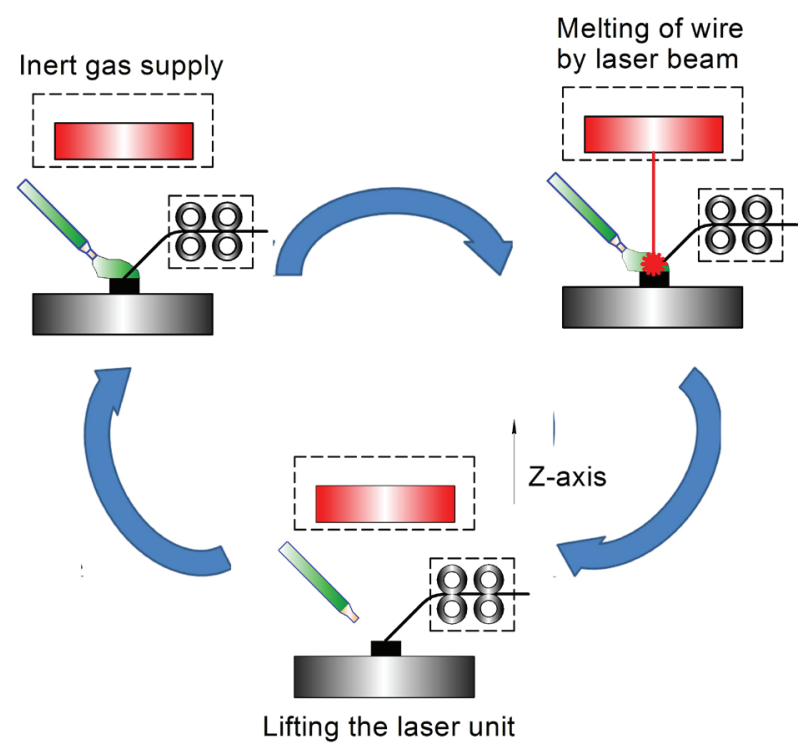

Fig. 2. The main steps of the LENS-w process.

\section{Results}

The results of mechanical pull tests of the samples manufactured using HTS 300 Mobile unit and made from $07 X 18$ H10T grade stainless steel wire are shown in Table 1 in comparison with reference properties of the material. 
Table 1. Parameters of laser irradiation.

\begin{tabular}{|c|c|c|}
\hline Sample \# & $\begin{array}{c}\text { Rupture } \\
\text { strength, } \\
\text { MPa }\end{array}$ & Tensile strain, $\boldsymbol{\varepsilon}_{\mathbf{t}} \mathbf{\%}$ \\
\hline 1 & 487 & 15 \\
\hline 2 & 594 & 24 \\
\hline Rod & 590 & 20 \\
\hline
\end{tabular}

Based on the tests results it is apparent that the mechanical properties of the samples made with HTS 300 Mobile unit and made from 07X18H10T grade stainless steel wire are comparable with the mechanical properties of the material supplied as rods.

In order to study microstructure of the samples they have performed fractographic investigations using the method of scanning-electron microscopy (Fig. 3).
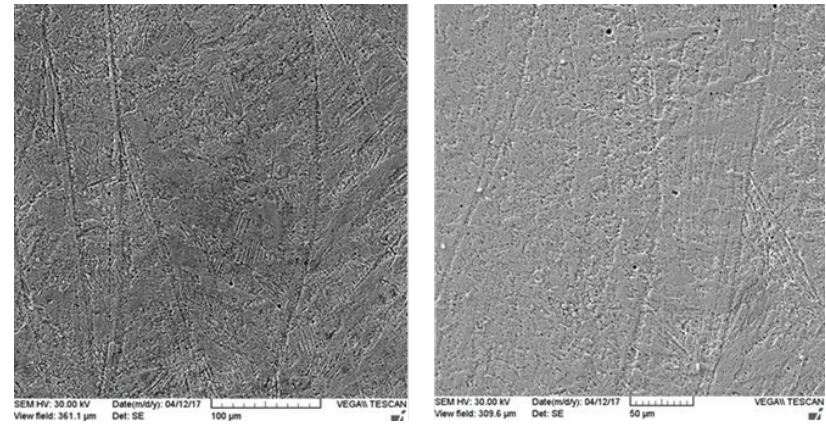

Fig. 3. Microstructure of the samples manufactured using HTS 300 Mobile unit and made of 07X18H10T grade stainless steel wire.

As shown in Fig. 3 the material's structure contains insignificant defects in the form of porosity with the diameter of less than $10 \mu \mathrm{m}$. There is no incomplete fusion and there are no cracks. Sandwich structure of the material is observed. The borders are clear and there is metallic linkage.

In order to study the borders between weld pads they have developed a map of allocation of the chemical elements in the microsection area (Fig. 4).

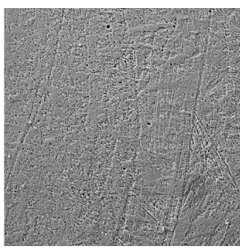

a)

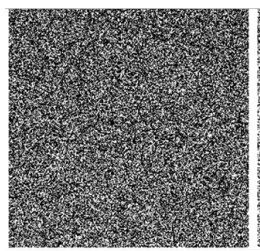

d)

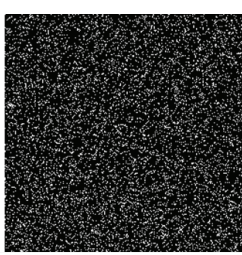

b)

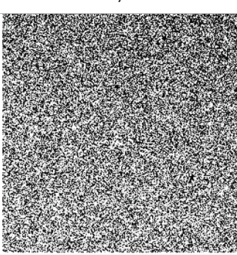

e)

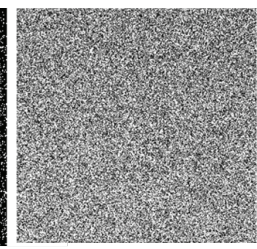

c)

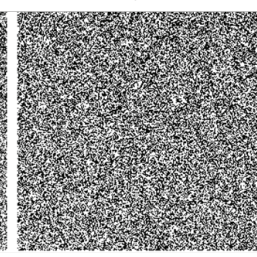

Fig. 4. Map of allocation of chemical elements

a) Electronic image of the microsection (x500); b) allocation of $\mathrm{Si}$; c) allocation of $\mathrm{Cr}$; d) allocation of $\mathrm{Mn}$; e) allocation of $\mathrm{Fe}$; f) allocation of $\mathrm{Ni}$
As shown in Fig. 3 in the area between weld pads there was a clear cut borderline of allocation of chemical elements. This fact also suggests that the wire has not been sufficiently fused in the process of overlay welding.

\section{Conclusion}

The test the structural layout of the direct laser alloying unit has been conducted. Samples made of 20X13 grade stainless steel wire have been manufactured using 07X18H10T grade stainless steel. In order to manufacture the samples HTS 300 Mobile unit has been employed.

The tested installation scheme can be used for the manufacture of parts such as full-sized test plates for pull testing materials.

The samples thus manufactured have the shatter- and moldability indices which are comparable with those of reference properties of the material when supplied as rods.

The porosity with the diameter of up10 $\mu \mathrm{m}$ as identified on the surface of a polished section as a result of the metallographic tests is apparently inherited, and it was present in the source material - 07X18H10T grade stainless steel wire.

The analysis of microstructure of the samples showed the presence of a clear cut borderline between the weld pads which is also validated by the allocation maps of chemical elements.

Influence of the parameters of impulse laser irradiation on fusibility of planes needs to be further researched, as well as their influence on the mechanical properties of materials.

This work was supported -1098.2017.8) and by the Ministry of Education and Science of the Russian Federation (Grant № 9.1299.2017) and partly in the framework of the implementation of the Program "Research and development on priority directions of scientific-technological complex of Russia for 2014 - 2020".

\section{References}

1. T. Caffrey and T. Wohlers: Wohlers Report 2015: Additive Manufacturing and 3D Printing State of the Industry (2015)

2. A.V. Agapovichev, A.V. Balaykin and V.G. Smelov, Modern Applied Science. 9 (2015)

3. V.G. Smelov, A.V. Sotov and A.V. Agapovichev, IOP Conf. Series: Materials Science and Engineering, 140 (2016)

4. V.Sh. Sufiyarov, A.A. Popovich, E.V. Borisov and I.A. Polozov, Tsvetnye Metally $=$ Non-ferrous metals, 1 (2015)

5. R. A. Vdovin and V.G. Smelov, J. of Engineering and Technology (IJET) 6, 5 (2014)

6. A.V. Balaykin, A.I. Kondratev and N.G. Galkina, J. Key Engineering Materials 684 (2016)

7. G. N. Levy, R. Schindel, and J.-P. Kruth, CIRP Annals - Manufacturing Technology, 52, 2 (2003) 
8. Carl Zeiss Jena GmbH: Additive Manufacturing at ZEISS, (2015)

9. V.G. Smelov, A.V. Sotov, A.V. Agapovichev and T.M. Tomilina, IOP Conf. Series: Materials Science and Engineering 142 (2016)

10. E. Walach, B. Widrow, IEEE Transactions on Information Theory, 20, 2 (1984)

11. A.B. Spierings, K.Wegener and G. Levy, Solid Freeform Fabrication Symposium SFF, Austin, TX, USA, (2012)

12. G. Kool and E. Amsterdam, The Future of Gas Turbine Technology - 5th International Conference, (2010)

13. K.S. Munir, Y. Li and C. Wen, Metallic Foam Bone: Processing, Modification and Characterization and Properties (2016)

14. V.G. Smelov, A.V. Sotov and A.V. Agapovichev, J. Key Engineering Materials 684 (2016) 